Proyecciones Journal of Mathematics

Vol. 38, No 2, pp. 267-293, June 2019.

Universidad Católica del Norte

Antofagasta - Chile

\title{
Further inequalities for log-convex functions related to Hermite-Hadamard result
}

\author{
S. S. Dragomir \\ Victoria University, Australia \\ Received: July 2017. Accepted : December 2018
}

\begin{abstract}
Some unweighted and weighted inequalities of Hermite-Hadamard type for log-convex functions defined on real intervals are given.
\end{abstract}

Subjclass: 26D15; 25D10.

Keywords: Convex functions, Integral inequalities, Log-Convex functions. 


\section{Introduction}

A function $f: I \rightarrow(0, \infty)$ is said to be log-convex or multiplicatively convex if $\log f$ is convex, or, equivalently, if for all $x, y \in I$ and $t \in[0,1]$ one has the inequality:

$$
f(t x+(1-t) y) \leq[f(x)]^{t}[f(y)]^{1-t} .
$$

We note that if $f$ and $g$ are convex and $g$ is increasing, then $g \circ f$ is convex; moreover, since $f=\exp (\log f)$, it follows that a log-convex function is convex, but the converse may not necessarily be true. This follows directly from (1.1) because, by the arithmetic-geometric mean inequality, we have

$$
[f(x)]^{t}[f(y)]^{1-t} \leq t f(x)+(1-t) f(y)
$$

for all $x, y \in I$ and $t \in[0,1]$.

Let us recall the Hermite-Hadamard inequality

$$
f\left(\frac{a+b}{2}\right) \leq \frac{1}{b-a} \int_{a}^{b} f(x) d x \leq \frac{f(a)+f(b)}{2},
$$

where $f: I \subseteq \mathbf{R} \rightarrow \mathbf{R}$ is a convex function on the interval $I, a, b \in I$ and $a<b$.

For related results, see [1]-[22], [25]-[29], [30]-[40] and [41]-[53].

Note that if we apply the above inequality for the log-convex functions $f: I \rightarrow(0, \infty)$, we have that

$$
\ln \left[f\left(\frac{a+b}{2}\right)\right] \leq \frac{1}{b-a} \int_{a}^{b} \ln f(x) d x \leq \frac{\ln f(a)+\ln f(b)}{2},
$$

from which we get

$$
f\left(\frac{a+b}{2}\right) \leq \exp \left[\frac{1}{b-a} \int_{a}^{b} \ln f(x) d x\right] \leq \sqrt{f(a) f(b)}
$$

that is an inequality of Hermite-Hadamard's type for log-convex functions.

By using simple properties of log-convex functions Dragomir and Mond proved in 1998 the following result [32].

Theorem 1. Let $f: I \rightarrow(0, \infty)$ be a log-convex mapping on $I$ and $a, b \in I$ with $a<b$. Then one has the inequality: 


$$
f\left(\frac{a+b}{2}\right) \leq \frac{1}{b-a} \int_{a}^{b} \sqrt{f(x) f(a+b-x)} d x \leq \sqrt{f(a) f(b)}
$$

The inequality between the first and second term in (1.5) may be improved as follows [32]. A different upper bound for the middle term in (1.5) can be also provided.

Theorem 2. Let $f: I \rightarrow(0, \infty)$ be a log-convex mapping on $I$ and $a, b \in I$ with $a<b$. Then one has the inequalities:

$$
\begin{aligned}
& f\left(\frac{a+b}{2}\right) \leq \exp \left[\frac{1}{b-a} \int_{a}^{b} \ln f(x) d x\right] \\
& \quad \leq \frac{1}{b-a} \int_{a}^{b} \sqrt{f(x) f(a+b-x)} d x \\
& \quad \leq \frac{1}{b-a} \int_{a}^{b} f(x) d x \leq L(f(a), f(b)),
\end{aligned}
$$

where $L(p, q)$ is the logarithmic mean of the strictly positive real numbers $p, q$, i.e.,

$$
L(p, q):=\frac{p-q}{\ln p-\ln q} \text { if } p \neq q \text { and } L(p, p):=p .
$$

The last inequality in (1.6) was obtained in a different context in [43]. As shown in [59], the following result also holds:

Theorem 3. Let $f: I \rightarrow(0, \infty)$ be a log-convex mapping on $I$ and $a, b \in I$ with $a<b$. Then one has the inequalities:

$$
f\left(\frac{a+b}{2}\right) \leq\left(\frac{1}{b-a} \int_{a}^{b} \sqrt{f(x)} d x\right)^{2} \leq \frac{1}{b-a} \int_{a}^{b} f(x) d x .
$$

We define the p-logarithmic mean as

$$
L_{p}(a, b):=\left\{\begin{array}{l}
{\left[\frac{b^{p+1}-a^{p+1}}{(p+1)(b-a)}\right]^{\frac{1}{p}}, \text { with } a \neq b} \\
a, \text { if } a=b
\end{array}\right.
$$

for $p \neq 0,-1$ and $a, b>0$.

In the recent work [28] we generalized the inequality (1.6) as follows: 
Theorem 4. Let $f:[a, b] \rightarrow(0, \infty)$ be a log-convex function on $[a, b]$.

Then for any $p>0$ we have the inequality

$$
f\left(\frac{a+b}{2}\right) \leq \exp \left[\frac{1}{b-a} \int_{a}^{b} \ln f(x) d x\right]
$$

$$
\begin{aligned}
& \leq\left(\frac{1}{b-a} \int_{a}^{b} f^{p}(x) f^{p}(a+b-x) d x\right)^{\frac{1}{2 p}} \\
& \leq\left(\frac{1}{b-a} \int_{a}^{b} f^{2 p}(x) d x\right)^{\frac{1}{2 p}} \\
& \leq\left\{\begin{array}{l}
{\left[L_{2 p-1}(f(a), f(b))\right]^{1-\frac{1}{2 p}}[L(f(a), f(b))]^{\frac{1}{2 p}}, p \neq \frac{1}{2}} \\
L(f(a), f(b)), p=\frac{1}{2} .
\end{array}\right.
\end{aligned}
$$

If $p \in\left(0, \frac{1}{2}\right)$, then we have

$$
\begin{aligned}
f\left(\frac{a+b}{2}\right) \leq \exp \left[\frac{1}{b-a} \int_{a}^{b} \ln f(x) d x\right] \\
\leq\left(\frac{1}{b-a} \int_{a}^{b} f^{p}(x) f^{p}(a+b-x) d x\right)^{\frac{1}{2 p}} \\
\leq\left(\frac{1}{b-a} \int_{a}^{b} f^{2 p}(x) d x\right)^{\frac{1}{2 p}} \leq \frac{1}{b-a} \int_{a}^{b} f(x) d x .
\end{aligned}
$$

Remark 1. If we take in (1.8) $p=1$, then we get

$$
\begin{aligned}
& f\left(\frac{a+b}{2}\right) \leq \exp \left[\frac{1}{b-a} \int_{a}^{b} \ln f(x) d x\right] \\
& \leq \quad\left(\frac{1}{b-a} \int_{a}^{b} f(x) f(a+b-x) d x\right)^{\frac{1}{2}} \\
& \leq\left(\frac{1}{b-a} \int_{a}^{b} f^{2}(x) d x\right)^{\frac{1}{2}} \\
& \leq \quad[A(f(a), f(b))]^{\frac{1}{2}}[L(f(a), f(b))]^{\frac{1}{2}} .
\end{aligned}
$$


If we take $p=\frac{1}{4}$ in (1.9), then we get

$$
\begin{aligned}
f\left(\frac{a+b}{2}\right) \leq \exp \left[\frac{1}{b-a} \int_{a}^{b} \ln f(x) d x\right] \\
\leq\left(\frac{1}{b-a} \int_{a}^{b} \sqrt[4]{f(x) f(a+b-x) d x}\right)^{2} \\
\leq\left(\frac{1}{b-a} \int_{a}^{b} \sqrt{f(x)} d x\right)^{2} \leq \frac{1}{b-a} \int_{a}^{b} f(x) d x .
\end{aligned}
$$

This improves the inequality (1.7).

Motivated by the above results, we establish in this paper some new unweighted and weighted inequalities for log-convex functions, some of them improving earlier results. Applications for special means are also provided.

\section{New Results}

The following result holds.

Theorem 1. Let $f:[a, b] \rightarrow(0, \infty)$ be a log-convex function. Then for every $t \in[0,1]$ we have

$$
\begin{gathered}
\int_{a}^{b} f(x) d x \geq \int_{a}^{b}[f(x)]^{1-t}[f(a+b-x)]^{t} d x \\
\geq\left\{\begin{array}{c}
\frac{1}{1-2 t} \int_{(1-t) a+t b}^{t a+(1-t) b} f(u) d u, \text { if } t \neq \frac{1}{2} \\
(b-a) f\left(\frac{a+b}{2}\right), \text { if } t=\frac{1}{2} .
\end{array}\right.
\end{gathered}
$$

Proof. The cases $t=0, \frac{1}{2}, 1$ are obvious.

Assume that $t \in(0,1) \backslash\left\{\frac{1}{2}\right\}$. By the log-convexity of $f$ we have

$$
\begin{gathered}
{[f(x)]^{1-t}[f(a+b-x)]^{t} \geq f((1-t) x+t(a+b-x))} \\
=f((1-2 t) x+t(a+b))
\end{gathered}
$$

for any $x \in[a, b]$. 
Integrating the inequality (2.2) over $x$ on $[a, b]$ we have

$$
\int_{a}^{b}[f(x)]^{1-t}[f(a+b-x)]^{t} d x \geq \int_{a}^{b} f((1-2 t) x+t(a+b)) d x .
$$

Since $t \neq \frac{1}{2}$, then $u:=(1-2 t) x+t(a+b)$ is a change of variable with $d u=(1-2 t) d x$.

For $x=a$ we get $u=(1-t) a+t b$ and for $x=b$ we get $u=t a+(1-t) b$. Therefore

$$
\int_{a}^{b} f((1-2 t) x+t(a+b)) d x=\frac{1}{1-2 t} \int_{(1-t) a+t b}^{t a+(1-t) b} f(u) d u
$$

and the second inequality in (2.1) is proved.

By the Hölder integral inequality for $p=\frac{1}{1-t}, q=\frac{1}{t}$ we have

$$
\begin{aligned}
& \int_{a}^{b}[f(x)]^{1-t}[f(a+b-x)]^{t} d x \\
& \leq\left(\int_{a}^{b}\left([f(x)]^{1-t}\right)^{\frac{1}{1-t}} d x\right)^{1-t}\left(\int_{a}^{b}\left([f(a+b-x)]^{t}\right)^{\frac{1}{t}} d x\right)^{t} \\
& =\left(\int_{a}^{b} f(x) d x\right)^{1-t}\left(\int_{a}^{b} f(a+b-x) d x\right)^{t} \\
& =\left(\int_{a}^{b} f(x) d x\right)^{1-t}\left(\int_{a}^{b} f(x) d x\right)^{t}=\int_{a}^{b} f(x) d x,
\end{aligned}
$$

that proves the first inequality in (2.1).

Corollary 1. Let $f:[a, b] \rightarrow(0, \infty)$ be a log-convex function. Then for every $t \in[0,1] \backslash\left\{\frac{1}{2}\right\}$ we have for $p>0$ that

$$
\left(\frac{1}{b-a} \int_{a}^{b} f^{2 p}(x) d x\right)^{\frac{1}{2 p}}
$$




$$
\begin{aligned}
& \geq\left(\frac{1}{b-a} \int_{a}^{b}\left[f^{2 p}(x)\right]^{1-t}\left[f^{2 p}(a+b-x)\right]^{t} d x\right)^{\frac{1}{2 p}} \\
& \geq\left(\frac{1}{(1-2 t)(b-a)} \int_{(1-t) a+t b}^{t a+(1-t) b} f^{2 p}(u) d u\right)^{\frac{1}{2 p}} \\
& \geq\left(\frac{1}{(1-2 t)(b-a)} \int_{(1-t) a+t b}^{t a+(1-t) b} f^{p}(u) f^{p}(a+b-u) d x\right)^{\frac{1}{2 p}} \\
& \geq \exp \left[\frac{1}{(1-2 t)(b-a)} \int_{(1-t) a+t b}^{t(1-t) b} \ln f(u) d u\right] \geq f\left(\frac{a+b}{2}\right) .
\end{aligned}
$$

Proof. Follows from Theorem 5 applied for the log-convex function $f^{2 p}$ with $p>0$ and by Theorem 4 applied for the interval $[(1-t) a+t b, t a+(1-t) b]$ when $t \in\left(0, \frac{1}{2}\right)$ or $\left.\left.[t a+(1-t) b,(1-t) a+t b]\right)\right)$ when $\left.t \in\left(\frac{1}{2}, 1\right)\right)$.

If we take $p=1 \mathrm{in}(2.3)$, then we get

$$
\left(\frac{1}{b-a} \int_{a}^{b} f^{2}(x) d x\right)^{\frac{1}{2}} \geq\left(\frac{1}{b-a} \int_{a}^{b}\left[f^{2}(x)\right]^{1-t}\left[f^{2}(a+b-x)\right]^{t} d x\right)^{\frac{1}{2}}
$$

$$
\begin{aligned}
& \geq\left(\frac{1}{(1-2 t)(b-a)} \int_{(1-t) a+t b}^{t a+(1-t) b} f^{2}(u) d u\right)^{\frac{1}{2}} \\
& \geq\left(\frac{1}{(1-2 t)(b-a)} \int_{(1-t) a+t b}^{t a+(1-t) b} f(u) f(a+b-u) d x\right)^{\frac{1}{2}} \\
& \geq \exp \left[\frac{1}{(1-2 t)(b-a)} \int_{(1-t) a+t b}^{t(1-t) b} \ln f(u) d u\right] \geq f\left(\frac{a+b}{2}\right) .
\end{aligned}
$$

If we take $p=\frac{1}{2}$ in $(2.3)$, then we get

$$
\frac{1}{b-a} \int_{a}^{b} f(x) d x
$$




$$
\begin{aligned}
& \geq \frac{1}{b-a} \int_{a}^{b}[f(x)]^{1-t}[f(a+b-x)]^{t} d x \\
& \geq \frac{1}{(1-2 t)(b-a)} \int_{(1-t) a+t b}^{t+(1-t) b} f(u) d u \\
& \geq \frac{1}{(1-2 t)(b-a)} \int_{(1-t) a+t b}^{t a+(1-t) b} \sqrt{f(u) f(a+b-u)} d x \\
& \geq \exp \left[\frac{1}{(1-2 t)(b-a)} \int_{(1-t) a+t b}^{t a+t} \ln f(u) d u\right] \geq f\left(\frac{a+b}{2}\right) .
\end{aligned}
$$

Corollary 2. Let $f:[a, b] \rightarrow(0, \infty)$ be a log-convex function. Then for every $t \in[0,1] \backslash\left\{\frac{1}{2}\right\}$ we have for $p \in\left(0, \frac{1}{2}\right)$ that

$$
\begin{gathered}
\frac{1}{b-a} \int_{a}^{b} f(x) d x \\
\geq \frac{1}{b-a} \int_{a}^{b}[f(x)]^{1-t}[f(a+b-x)]^{t} d x \\
\geq \frac{1}{t a+(1-t) b} \int_{(1-t) a+t b}^{t(b-a)} f(u) d u \\
\geq\left(\frac{1}{\left.\frac{t a+(1-t) b}{(1-2 t)(b-a)} \int_{(1-t) a+t b}^{t a}(u) d u\right)^{\frac{1}{2 p}}} \int^{t a+(1-t) b} \int_{(1-t) a+t b}^{t a+(1-t) b} f^{p}(u) f^{p}(a+b-u) d u\right)^{\frac{1}{2 p}} \\
\geq \operatorname{exp~}\left[\frac{1}{(1-2 t)(b-a)} \int_{(1-t) a+t b}^{1} \ln f(u) d u\right] \geq f\left(\frac{a+b}{2}\right) .
\end{gathered}
$$

Follows by Theorem 5 and Theorem 4 for $p \in\left(0, \frac{1}{2}\right)$.

If we take $p=\frac{1}{4}$ in $(2.6)$, then we get

$$
\frac{1}{b-a} \int_{a}^{b} f(x) d x
$$




$$
\begin{aligned}
& \geq \frac{1}{b-a} \int_{a}^{b}[f(x)]^{1-t}[f(a+b-x)]^{t} d x \\
& \geq \frac{1}{(1-2 t)(b-a)} \int_{(1-t) a+t b}^{t+t) b} f(u) d u \\
& \geq\left(\frac{1}{(1-2 t)(b-a)} \int_{(1-t) a+t b}^{t a+(1-t) b} \sqrt{f(u)} d u\right)^{2} \\
& \geq\left(\frac{1}{(1-2 t)(b-a)} \int_{(1-t) a+t b}^{t+t) b} \sqrt[4]{f(u) f(a+b-u)} d u\right)^{2} \\
& \geq \exp \left[\frac{1}{(1-2 t)(b-a+(1-t) b} \int_{(1-t) a+t b} \ln f(u) d u\right] \geq f\left(\frac{a+b}{2}\right) .
\end{aligned}
$$

If we use the inequality

$$
\frac{1}{b-a} \int_{a}^{b} f(x) d x \geq \frac{1}{(1-2 t)(b-a)} \int_{(1-t) a+t b}^{t a+(1-t) b} f(u) d u
$$

where $t \neq \frac{1}{2}$, for the log-convex function $f:[a, b] \rightarrow(0, \infty), f(x)=\frac{1}{x}$, then we get

$$
\frac{\ln b-\ln a}{b-a} \geq \frac{\ln (t a+(1-t) b)-\ln ((1-t) a+t b)}{(t a+(1-t) b)-(((1-t) a+t b))}
$$

which, in terms of logarithmic mean, is

$$
L(t a+(1-t) b,(1-t) a+t b) \geq L(a, b)
$$

for any $t \in[0,1] \backslash\left\{\frac{1}{2}\right\}$.

When $t=\frac{1}{2}$ the inequality $(2.9)$ becomes $A(a, b) \geq L(a, b)$ that is also true.

For $q \neq 0,-1$ define the $L_{q}$-logarithmic mean as

$$
L_{q}(a, b):=\left\{\begin{array}{c}
\left(\frac{b^{q+1}-a^{q+1}}{(q+1)(b-a)}\right)^{\frac{1}{q}} \text { if } b \neq a, \\
a \text { if } b=a .
\end{array}\right.
$$

If we use the inequality (2.8) for the log-convex function $f:[a, b] \rightarrow$ $(0, \infty), f(x)=\frac{1}{x^{p}}$, with $p>0, p \neq 1$, then we have for any $t \in[0,1] \backslash\left\{\frac{1}{2}\right\}$ that 


$$
\frac{1}{b-a} \int_{a}^{b} x^{-p} d x \geq \frac{1}{(1-2 t)(b-a)} \int_{(1-t) a+t b}^{t a+(1-t) b} u^{-p} d u
$$

i.e.

$$
\frac{b^{-p+1}-a^{-p+1}}{(1-p)(b-a)} \geq \frac{(t a+(1-t) b)^{-p+1}-((1-t) a+t b)^{-p+1}}{((1-p))[(t a+(1-t) b)-(((1-t) a+t b))]}
$$

which can be written as

$$
L_{-p}^{-p}(a, b) \geq L_{-p}^{-p}(t a+(1-t) b,(1-t) a+t b) .
$$

Therefore we have

$$
L_{-p}(t a+(1-t) b,(1-t) a+t b) \geq L_{-p}(a, b)
$$

for any $p>0, p \neq 1$ and for any $t \in[0,1] \backslash\left\{\frac{1}{2}\right\}$. The case $t=\frac{1}{2}$ reduces to the known inequality $A(a, b) \geq L_{-p}(a, b)$ for any $p>0$.

\section{Weighted Inequalities}

We have the following generalized weighted version of the inequality (1.5).

Theorem 1. Let $f:[a, b] \rightarrow(0, \infty)$ be a log-convex function. If $w:$ $[a, b] \rightarrow[0, \infty)$ is integrable and $\int_{a}^{b} w(x) d x>0$, then

(3.1) $f\left(\frac{a+b}{2}\right) \leq\left(\frac{\int_{a}^{b} w(x) f^{p}(x) f^{p}(a+b-x) d x}{\int_{a}^{b} w(x) d x}\right)^{\frac{1}{2 p}} \leq \sqrt{f(a) f(b)}$

for any $p>0$.

In particular, we have

$$
f\left(\frac{a+b}{2}\right) \leq\left(\frac{\int_{a}^{b} w(x) f(x) f(a+b-x) d x}{\int_{a}^{b} w(x) d x}\right)^{\frac{1}{2}} \leq \sqrt{f(a) f(b)}
$$


Proof. We know that, see [32] or [35, p. 198], if $g$ is log-convex, then

$$
g\left(\frac{a+b}{2}\right) \leq \sqrt{g(x) g(a+b-x)} \leq \sqrt{g(a) g(b)}
$$

for any $x \in[a, b]$.

For any $p>0$ the function $f^{2 p}$ is log-convex and by (3.3) we have

$$
f^{2 p}\left(\frac{a+b}{2}\right) \leq f^{p}(x) f^{p}(a+b-x) \leq f^{p}(a) f^{p}(b)
$$

for any $x \in[a, b]$.

If we multiply (3.4) by $w(x) \geq 0$ and integrate, then we get

$$
\begin{gathered}
f^{2 p}\left(\frac{a+b}{2}\right) \int_{a}^{b} w(x) d x \leq \int_{a}^{b} w(x) f^{p}(x) f^{p}(a+b-x) d x \\
\leq f^{p}(a) f^{p}(b) \int_{a}^{b} w(x) d x
\end{gathered}
$$

namely

$$
f^{2 p}\left(\frac{a+b}{2}\right) \leq \frac{\int_{a}^{b} w(x) f^{p}(x) f^{p}(a+b-x) d x}{\int_{a}^{b} w(x) d x} \leq f^{p}(a) f^{p}(b) .
$$

Taking the power $\frac{1}{2 p}$ in (3.5) we get the desired result (3.1).

We also have the inequality

$$
f\left(\frac{a+b}{2}\right) \leq \frac{\int_{a}^{b} w(x) \sqrt{f(x) f(a+b-x)} d x}{\int_{a}^{b} w(x) d x} \leq \sqrt{f(a) f(b)},
$$

that is a weighted version of (1.5).

If we take $p=\frac{1}{4}$ in (3.1), then we get

$$
f\left(\frac{a+b}{2}\right) \leq\left(\frac{\int_{a}^{b} w(x) \sqrt[4]{f(x) f(a+b-x)} d x}{\int_{a}^{b} w(x) d x}\right)^{2} \leq \sqrt{f(a) f(b)}
$$

Using Jensen's inequality for the power $p \geq 1(p \in(0,1))$, namely

$$
\left(\frac{\int_{a}^{b} w(x) g(x) d x}{\int_{a}^{b} w(x) d x}\right)^{p} \leq(\geq) \frac{\int_{a}^{b} w(x) g^{p}(x) d x}{\int_{a}^{b} w(x) d x},
$$

we can start the following more precise result: 
Corollary 3. Let $f:[a, b] \rightarrow(0, \infty)$ be a log-convex function and $w$ : $[a, b] \rightarrow[0, \infty)$ be integrable and $\int_{a}^{b} w(x) d x>0$.

If $p \geq 1$, then

$$
\begin{gathered}
f\left(\frac{a+b}{2}\right) \leq\left(\frac{\int_{a}^{b} w(x) f(x) f(a+b-x) d x}{\int_{a}^{b} w(x) d x}\right)^{\frac{1}{2}} \\
\leq\left(\frac{\int_{a}^{b} w(x) f^{p}(x) f^{p}(a+b-x) d x}{\int_{a}^{b} w(x) d x}\right)^{\frac{1}{2 p}} \leq \sqrt{f(a) f(b)} .
\end{gathered}
$$

If $p \in(0,1)$, then

$$
\begin{aligned}
& f\left(\frac{a+b}{2}\right) \leq\left(\frac{\int_{a}^{b} w(x) f^{p}(x) f^{p}(a+b-x) d x}{\int_{a}^{b} w(x) d x}\right)^{\frac{1}{2 p}} \\
& \leq\left(\frac{\int_{a}^{b} w(x) f(x) f(a+b-x) d x}{\int_{a}^{b} w(x) d x}\right)^{\frac{1}{2}} \leq \sqrt{f(a) f(b)} .
\end{aligned}
$$

Remark 2. Let $f:[a, b] \rightarrow(0, \infty)$ be a log-convex function. We observe that if we take in (3.1) $w(x)=f^{-p}(a+b-x), p>0$, then we get

$$
f\left(\frac{a+b}{2}\right) \leq\left(\frac{\int_{a}^{b} f^{p}(x) d x}{\int_{a}^{b} f^{-p}(x) d x}\right)^{\frac{1}{2 p}} \leq \sqrt{f(a) f(b)}
$$

for any $p>0$.

In particular, we have the inequalities

$$
f\left(\frac{a+b}{2}\right) \leq\left(\frac{\int_{a}^{b} f(x) d x}{\int_{a}^{b} \frac{1}{f(x)} d x}\right)^{\frac{1}{2}} \leq \sqrt{f(a) f(b)}
$$

and

$$
f\left(\frac{a+b}{2}\right) \leq \frac{\int_{a}^{b} \sqrt{f(x)} d x}{\int_{a}^{b} \frac{1}{\sqrt{f(x)}} d x} \leq \sqrt{f(a) f(b)} .
$$

If we take in (3.10) $f(x)=x^{-1}, x \in[a, b] \subset(0, \infty)$, which is log-convex, then we have 


$$
\left(\frac{a+b}{2}\right)^{-1} \leq\left(\frac{\int_{a}^{b} x^{-p} d x}{\int_{a}^{b} x^{p} d x}\right)^{\frac{1}{2 p}} \leq(\sqrt{f(a) f(b)})^{-1}
$$

Observe that

$$
\begin{aligned}
\frac{\int_{a}^{b} x^{-p} d x}{\int_{a}^{b} x^{p} d x} & =\frac{\frac{1}{b-a} \int_{a}^{b} x^{-p} d x}{\frac{1}{b-a} \int_{a}^{b} x^{p} d x} \\
& =\frac{\left[L_{-p}(a, b)\right]^{-p}}{\left[L_{p}(a, b)\right]^{p}}
\end{aligned}
$$

and by (3.13) we have

$$
\left(\frac{a+b}{2}\right)^{-1} \leq\left(\frac{1}{\left[L_{p}(a, b)\right]\left[L_{-p}(a, b)\right]}\right)^{\frac{1}{2}} \leq(\sqrt{f(a) f(b)})^{-1}
$$

that is equivalent to

$$
G(a, b) \leq \sqrt{\left[L_{p}(a, b)\right]\left[L_{-p}(a, b)\right]} \leq A(a, b) .
$$

This can be also written as

$$
G(a, b) \leq G\left(L_{p}(a, b), L_{-p}(a, b)\right) \leq A(a, b) .
$$

If we take in the first inequality (3.14) $p=1$, then we get

$$
G^{2}(a, b) \leq A(a, b) L(a, b) .
$$

We have the following weighted version of (1.4).

Theorem 2. Let $f:[a, b] \rightarrow(0, \infty)$ be a log-convex function. If $w:$ $[a, b] \rightarrow[0, \infty)$ is integrable and $\int_{a}^{b} w(x) d x>0$, then

$$
\begin{aligned}
& f\left(\frac{\int_{a}^{b} w(x) x d x}{\int_{a}^{b} w(x) d x}\right) \\
& \leq \exp \left(\frac{\int_{a}^{b} w(x) \ln f(x) d x}{\int_{a}^{b} w(x) d x}\right) \\
& \leq[f(b)]^{\frac{1}{b-a}}\left(\frac{\int_{a}^{b} x w(x) d x}{\int_{a}^{b} w(x) d x}-a\right)[f(a)]^{\frac{1}{b-a}}\left(b-\frac{\int_{a}^{b} x w(x) d x}{\int_{a}^{b} w(x) d x}\right) \\
& \leq \frac{1}{b-a}\left[\left(\frac{\int_{a}^{b} x w(x) d x}{\int_{a}^{b} w(x) d x}-a\right) f(b)+\left(b-\frac{\int_{a}^{b} x w(x) d x}{\int_{a}^{b} w(x) d x}\right) f(a)\right] \text {. }
\end{aligned}
$$


Proof. Since $\ln f$ is convex, then by Jensen's inequality we have

$$
\ln f\left(\frac{\int_{a}^{b} w(x) x d x}{\int_{a}^{b} w(x) d x}\right) \leq \frac{\int_{a}^{b} w(x) \ln f(x) d x}{\int_{a}^{b} w(x) d x} .
$$

Taking the exponential in (3.17) we get the first inequality in (3.16).

Since $\ln f$ is convex, then

$\ln f(x)=\ln f\left(\frac{x-a}{b-a} b+\frac{b-x}{b-a} a\right) \leq \frac{x-a}{b-a} \ln f(b)+\frac{b-x}{b-a} \ln f(a)$

for any $x \in[a, b]$.

By taking the weighted integral mean in (3.18) we get

$$
\begin{gathered}
\frac{\int_{a}^{b} w(x) \ln f(x) d x}{\int_{a}^{b} w(x) d x} \\
\leq \frac{1}{b-a}\left[\left(\frac{\int_{a}^{b} x w(x) d x}{\int_{a}^{b} w(x) d x}-a\right) \ln f(b)+\left(b-\frac{\int_{a}^{b} x w(x) d x}{\int_{a}^{b} w(x) d x}\right) \ln f(a)\right] \\
=\ln \left([f(b)]^{\frac{1}{b-a}\left(\frac{\int_{a}^{b} x w(x) d x}{\int_{a}^{b} w(x) d x}-a\right)}[f(a)]^{\frac{1}{b-a}\left(b-\frac{\int_{a}^{b} x w(x) d x}{\int_{a}^{b} w(x) d x}\right)}\right) .
\end{gathered}
$$

By taking the exponential in (3.19), we get the second inequality in (3.16).

The last part of (3.16) follows by the weighted geometric mean-arithmetic mean inequality.

Remark 3. If we take $w(x)=1, x \in[a, b]$ in the first two inequalities (3.16), we recapture (1.4).

If we take $w(x)=\frac{1}{x}, x \in[a, b] \subset(0, \infty)$ in (3.16), then we get

$$
\begin{aligned}
& f(L(a, b)) \leq \exp \left(\frac{1}{\ln b-\ln a} \int_{a}^{b} \frac{\ln f(x)}{x} d x\right) \\
\leq & {[f(b)]^{\frac{L(a, b)-a}{b-a}}[f(a)]^{\frac{b-L(a, b)}{b-a}} } \\
\leq & \frac{(L(a, b)-a) f(b)+(b-L(a, b)) f(a)}{b-a},
\end{aligned}
$$


where $L(a, b)$ is the logarithmic mean, i.e.

$$
L(a, b)=\left\{\begin{array}{l}
\frac{b-a}{\ln b-\ln a} \text { if } a \neq b \\
a \text { if } a=b .
\end{array}\right.
$$

If we take $w(x)=\frac{1}{x^{2}}, x \in[a, b] \subset(0, \infty)$ in (3.16), then we get

$$
\begin{aligned}
& f\left(\frac{G^{2}(a, b)}{L(a, b)}\right) \leq \exp \left(\frac{a b}{b-a} \int_{a}^{b} \frac{\ln f(x)}{x^{2}} d x\right) \\
\leq & {[f(b)]^{\frac{1}{b-a}\left(\frac{G^{2}(a, b)}{L(a, b)}-a\right)}[f(a)]^{\frac{1}{b-a}\left(b-\frac{G^{2}(a, b)}{L(a, b)}\right)} } \\
\leq & \frac{1}{b-a}\left[\left(\frac{G^{2}(a, b)}{L(a, b)}-a\right) f(b)+\left(b-\frac{G^{2}(a, b)}{L(a, b)}\right) f(a)\right] .
\end{aligned}
$$

We also have the alternative result:

Theorem 3. Let $f:[a, b] \rightarrow(0, \infty)$ be a log-convex function. If $w:$ $[a, b] \rightarrow[0, \infty)$ is integrable and $\int_{a}^{b} w(x) d x>0$,

then

$$
\begin{gathered}
f\left(\frac{\int_{a}^{b} w(x) x d x}{\int_{a}^{b} w(x) d x}\right) \\
\leq \exp \left(\frac{\int_{a}^{b} w(x) \ln f(x) d x}{\int_{a}^{b} w(x) d x}\right) \\
\leq \frac{\int_{a}^{b} w(x) f(x) d x}{\int_{a}^{b} w(x) d x} \leq\left(\frac{[f(a)]^{b}}{[f(b)]^{a}}\right)^{\frac{1}{b-a}} \frac{\int_{a}^{b} w(x)\left(\frac{f(b)}{f(a)}\right)^{\frac{x}{b-a}} d x}{\int_{a}^{b} w(x) d x} \\
\leq \frac{1}{b-a}\left[\left(\frac{\int_{a}^{b} x w(x) d x}{\int_{a}^{b} w(x) d x}-a\right) f(b)+\left(b-\frac{\int_{a}^{b} x w(x) d x}{\int_{a}^{b} w(x) d x}\right) f(a)\right] .
\end{gathered}
$$

Proof. Using Jensen's inequality for the exponential function we have

$$
\begin{aligned}
\exp \left(\frac{\int_{a}^{b} w(x) \ln f(x) d x}{\int_{a}^{b} w(x) d x}\right) & \leq \frac{\int_{a}^{b} w(x) \exp (\ln f(x)) d x}{\int_{a}^{b} w(x) d x} \\
& =\frac{\int_{a}^{b} w(x) f(x) d x}{\int_{a}^{b} w(x) d x}
\end{aligned}
$$

and the second inequality in (3.22) is proved.

From (3.18) and the arithmetic mean - geometric mean inequality we have 


$$
\begin{gathered}
f(x) \leq[f(b)]^{\frac{x-a}{b-a}}[f(a)]^{\frac{b-x}{b-a}}=\left(\frac{[f(a)]^{b}}{[f(b)]^{a}}\right)^{\frac{1}{b-a}}\left(\frac{f(b)}{f(a)}\right)^{\frac{x}{b-a}} \\
\leq \frac{x-a}{b-a} f(b)+\frac{b-x}{b-a} f(a)
\end{gathered}
$$

for any $x \in[a, b]$.

By taking the weighted integral mean in (3.23) we get

$$
\begin{aligned}
& \frac{\int_{a}^{b} w(x) f(x) d x}{\int_{a}^{b} w(x) d x} \\
& \leq\left(\frac{[f(a)]^{b}}{[f(b)]^{a}}\right)^{\frac{1}{b-a}} \frac{\int_{a}^{b} w(x)\left(\frac{f(b)}{f(a)}\right)^{\frac{x}{b-a}} d x}{\int_{a}^{b} w(x) d x} \\
& \leq \frac{1}{b-a}\left[\left(\frac{\int_{a}^{b} x w(x) d x}{\int_{a}^{b} w(x) d x}-a\right) f(b)+\left(b-\frac{\int_{a}^{b} x w(x) d x}{\int_{a}^{b} w(x) d x}\right) f(a)\right]
\end{aligned}
$$

and the last part of (3.22) is proved.

Remark 4. If we take $w(x)=1, x \in[a, b]$ in (3.22), then we have

$$
f\left(\frac{a+b}{2}\right) \leq \exp \left(\frac{1}{b-a} \int_{a}^{b} \ln f(x) d x\right)
$$

$\leq \frac{1}{b-a} \int_{a}^{b} f(x) d x \leq L(f(a), f(b)) \leq \frac{f(a)+f(b)}{2}$.

If we take $w(x)=\frac{1}{x}, x \in[a, b] \subset(0, \infty)$ in (3.22), then we get

$$
\begin{aligned}
& f(L(a, b)) \leq \exp \left(\frac{1}{\ln b-\ln a} \int_{a}^{b} \frac{\ln f(x)}{x} d x\right) \\
\leq & \frac{1}{\ln b-\ln a} \int_{a}^{b} \frac{f(x)}{x} d x \\
\leq & \left(\frac{[f(a)]^{b}}{[f(b)]^{a}}\right)^{\frac{1}{b-a}} \frac{\int_{a}^{b} \frac{1}{x}\left(\frac{f(b)}{\ln (a)}\right)^{\frac{x}{b-a}} d x}{\ln b-\ln a} \\
\leq & \frac{(L(a, b)-a) f(b)+(b-L(a, b)) f(a)}{b-a} .
\end{aligned}
$$

\section{Inequalities for Symmetric Weights}

We say that the weight $w:[a, b] \rightarrow[0, \infty)$ is symmetric on $[a, b]$ if

$$
w(a+b-x)=w(x) \text { for all } x \in[a, b] .
$$


It is well known that if $f:[a, b] \rightarrow \mathbf{R}$ is convex and $w:[a, b] \rightarrow[0, \infty)$ is integrable and symmetric on $[a, b]$, then the Fejér inequality holds

$$
f\left(\frac{a+b}{2}\right) \leq \frac{\int_{a}^{b} w(x) f(x) d x}{\int_{a}^{b} w(x) d x} \leq \frac{f(a)+f(b)}{2} .
$$

If $f:[a, b] \rightarrow(0, \infty)$ is a log-convex function on $[a, b]$ and $w:[a, b] \rightarrow$ $[0, \infty)$ is integrable and symmetric on $[a, b]$, then by $(4.1)$ we have

$$
\ln f\left(\frac{a+b}{2}\right) \leq \frac{\int_{a}^{b} w(x) \ln f(x) d x}{\int_{a}^{b} w(x) d x} \leq \frac{\ln f(a)+\ln f(b)}{2},
$$

which is equivalent to

$$
f\left(\frac{a+b}{2}\right) \leq \exp \left(\frac{\int_{a}^{b} w(x) \ln f(x) d x}{\int_{a}^{b} w(x) d x}\right) \leq \sqrt{f(a) f(b)} .
$$

Theorem 1. Let $f:[a, b] \rightarrow(0, \infty)$ be a log-convex function on $[a, b]$ and $w:[a, b] \rightarrow[0, \infty)$ be integrable and symmetric on $[a, b]$. Then

$$
\begin{aligned}
& f\left(\frac{a+b}{2}\right) \leq \exp \left(\frac{\int_{a}^{b} w(x) \ln f(x) d x}{\int_{a}^{b} w(x) d x}\right) \\
& \leq \frac{\int_{a}^{b} w(x) \sqrt{f(x) f(a+b-x)} d x}{\int_{a}^{b} w(x) d x} \leq \frac{\int_{a}^{b} w(x) f(x) d x}{\int_{a}^{b} w(x) d x}
\end{aligned}
$$

Proof. By Jensen's integral inequality for the exponential we have

$$
\begin{aligned}
& \exp \left(\frac{\int_{a}^{b} w(x) \ln \sqrt{f(x) f(a+b-x)}}{\int_{a}^{b} w(x) d x}\right) \\
\leq & \frac{\int_{a}^{b} w(x) \exp (\ln \sqrt{f(x) f(a+b-x)}) d x}{\int_{a}^{b} w(x) d x} \\
= & \frac{\int_{a}^{b} w(x) \sqrt{f(x) f(a+b-x)} d x}{\int_{a}^{b} w(x) d x} .
\end{aligned}
$$

Observe, by the symmetry of $w$, that 


$$
\begin{aligned}
& \int_{a}^{b} w(x) \ln \sqrt{f(x) f(a+b-x)} d x \\
& =\frac{1}{2}\left[\int_{a}^{b} w(x) \ln f(x) d x+\int_{a}^{b} w(x) \ln f(a+b-x) d x\right] \\
& =\frac{1}{2}\left[\int_{a}^{b} w(x) \ln f(x) d x+\int_{a}^{b} w(a+b-x) \ln f(a+b-x) d x\right] \\
& =\int_{a}^{b} w(x) \ln f(x) d x
\end{aligned}
$$

since, obviously

$$
\int_{a}^{b} w(a+b-x) \ln f(a+b-x) d x=\int_{a}^{b} w(x) \ln f(x) d x .
$$

By (4.4) we then get the second inequality in (4.3).

By Cauchy-Buniakovski-Schwarz integral inequality we also have

$$
\begin{aligned}
& \int_{a}^{b} w(x) \sqrt{f(x) f(a+b-x)} d x \\
& \leq \sqrt{\int_{a}^{b} w(x) f(x) d x} \sqrt{\int_{a}^{b} w(x) f((a+b-x)) d x} \\
& =\sqrt{\int_{a}^{b} w(x) f(x) d x} \sqrt{\int_{a}^{b} w(x) f(x) d x} \\
& =\int_{a}^{b} w(x) f(x) d x,
\end{aligned}
$$

which proves the third inequality in (4.3).

The above inequality (4.2) may be generalized as follows by replacing $f$ with $f^{2 p}$ for $p>0$.

Corollary 4. Let $f:[a, b] \rightarrow(0, \infty)$ be a log-convex function on $[a, b]$ and $w:[a, b] \rightarrow[0, \infty)$ be integrable and symmetric on $[a, b]$. Then for any $p>0$ we have

$$
f\left(\frac{a+b}{2}\right) \leq \exp \left(\frac{\int_{a}^{b} w(x) \ln f(x) d x}{\int_{a}^{b} w(x) d x}\right)
$$




$$
\begin{aligned}
& \leq \\
& \left(\frac{\int_{a}^{b} w(x) f^{p}(x) f^{p}(a+b-x) d x}{\int_{a}^{b} w(x) d x}\right)^{\frac{1}{2 p}} \\
& \leq \\
& \left(\frac{\int_{a}^{b} w(x) f^{2 p}(x) d x}{\int_{a}^{b} w(x) d x}\right)^{\frac{1}{2 p}} .
\end{aligned}
$$

Remark 5. We observe that for $p \geq 1$ we have

$$
\begin{aligned}
& f\left(\frac{a+b}{2}\right) \leq \exp \left(\frac{\int_{a}^{b} w(x) \ln f(x) d x}{\int_{a}^{b} w(x) d x}\right) \\
\leq & \left(\frac{\int_{a}^{b} w(x) f(x) f(a+b-x) d x}{\int_{a}^{b} w(x) d x}\right)^{\frac{1}{2}} \\
\leq & \left(\frac{\int_{a}^{b} w(x) f^{p}(x) f^{p}(a+b-x) d x}{\int_{a}^{b} w(x) d x}\right)^{\frac{1}{2 p}} \\
\leq & \left(\frac{\int_{a}^{b} w(x) f^{2 p}(x) d x}{\int_{a}^{b} w(x) d x}\right)^{\frac{1}{2 p}}
\end{aligned}
$$

and for $p \in(0,1)$

$$
\begin{aligned}
& f\left(\frac{a+b}{2}\right) \leq \exp \left(\frac{\int_{a}^{b} w(x) \ln f(x) d x}{\int_{a}^{b} w(x) d x}\right) \\
\leq & \left(\frac{\int_{a}^{b} w(x) f^{p}(x) f^{p}(a+b-x) d x}{\int_{a}^{b} w(x) d x}\right)^{\frac{1}{2 p}} \\
\leq & \left(\frac{\int_{a}^{b} w(x) f(x) f(a+b-x) d x}{\int_{a}^{b} w(x) d x}\right)^{\frac{1}{2}} \\
\leq & \left(\frac{\int_{a}^{b} w(x) f^{2}(x) d x}{\int_{a}^{b} w(x) d x}\right)^{\frac{1}{2}} .
\end{aligned}
$$

Finally, we have: 
Theorem 2. Let $f:[a, b] \rightarrow(0, \infty)$ be a log-convex function on $[a, b]$ and $w:[a, b] \rightarrow[0, \infty)$ be integrable and symmetric on $[a, b]$. Then for any $p>0$ we have

$$
f\left(\frac{a+b}{2}\right) \leq\left(\frac{\int_{a}^{b} f^{p}(x) w(x) d x}{\int_{a}^{b} \frac{w(x) d x}{f^{p}(x)}}\right)^{\frac{1}{2 p}} \leq \sqrt{f(a) f(b)} .
$$

Proof. From (3.4) we have

$$
f^{2 p}\left(\frac{a+b}{2}\right) \frac{1}{f^{p}(a+b-x)} \leq f^{p}(x) \leq f^{p}(a) f^{p}(b) \frac{1}{f^{p}(a+b-x)}
$$

for any $x \in[a, b]$.

If we multiply by $w(x) \geq 0$ and integrate on $[a, b]$, then we get

$$
\begin{aligned}
& f^{2 p}\left(\frac{a+b}{2}\right) \int_{a}^{b} \frac{w(x) d x}{f^{p}(a+b-x)} \\
& \leq \int_{a}^{b} f^{p}(x) w(x) d x \\
& \leq f^{p}(a) f^{p}(b) \int_{a}^{b} \frac{w(x) d x}{f^{p}(a+b-x)} .
\end{aligned}
$$

Since, by symmetry of $w$ we have

$$
\int_{a}^{b} \frac{w(x) d x}{f^{p}(a+b-x)}=\int_{a}^{b} \frac{w(a+b-x) d x}{f^{p}(a+b-x)}=\int_{a}^{b} \frac{w(x) d x}{f^{p}(x)},
$$

which implies that

$$
\begin{aligned}
& f^{2 p}\left(\frac{a+b}{2}\right) \int_{a}^{b} \frac{w(x) d x}{f^{p}(x)} \\
& \leq \int_{a}^{b} f^{p}(x) w(x) d x \\
& \leq f^{p}(a) f^{p}(b) \int_{a}^{b} \frac{w(x) d x}{f^{p}(x)} .
\end{aligned}
$$

and the inequality (4.8) is proved.

Remark 6. If we write the inequality (4.8) for the log-convex function $f:[a, b] \subset(0, \infty) \rightarrow(0, \infty), f(x)=\frac{1}{x}$, then we have for $p>0$

$$
\left(\frac{a+b}{2}\right)^{-1} \leq\left(\frac{\int_{a}^{b} x^{-p} w(x) d x}{\int_{a}^{b} x^{p} w(x) d x}\right)^{\frac{1}{2 p}} \leq(\sqrt{a b})^{-1},
$$


that is equivalent to

$$
G(a, b) \leq\left(\frac{\int_{a}^{b} x^{p} w(x) d x}{\int_{a}^{b} x^{-p} w(x) d x}\right)^{\frac{1}{2 p}} \leq A(a, b),
$$

for any symmetric integrable weight $w:[a, b] \rightarrow[0, \infty)$.

\section{References}

[1] M. Alomari and M. Darus, The Hadamard's inequality for s-convex function. Int. J. Math. Anal. (Ruse) 2, No. 13-16, pp. 639-646, (2008).

[2] M. Alomari and M. Darus, Hadamard-type inequalities for s-convex functions. Int. Math. Forum 3, No. 37-40, pp. 1965-1975, (2008).

[3] G. A. Anastassiou, Univariate Ostrowski inequalities, revisited. Monatsh. Math., 135, No. 3, pp. 175-189, (2002).

[4] G. D. Anderson, M. K. Vamanamurthy and M. Vuorinen, Generalized convexity and inequalities, J. Math. Anal. Appl. 335, pp. 1294-1308, (2007).

[5] N. S. Barnett, P. Cerone, S. S. Dragomir, M. R. Pinheiro,and A. Sofo, Ostrowski type inequalities for functions whose modulus of the derivatives are convex and applications. Inequality Theory and Applications, Vol. 2 (Chinju/Masan, 2001), 19-32, Nova Sci. Publ., Hauppauge, NY, 2003. Preprint: RGMIA Res. Rep. Coll. 5, (2002), No. 2, Art. 1 [Online http://rgmia.org/papers/v5n2/Paperwapp2q.pdf].

[6] E. F. Beckenbach, Convex functions, Bull. Amer. Math. Soc. 54, pp. 439-460, (1948).

[7] M. Bombardelli and S. Varošanec, Properties of h-convex functions related to the Hermite-Hadamard-Fejér inequalities. Comput. Math. Appl. 58, No. 9, pp. 1869-1877, (2009).

[8] W. W. Breckner, Stetigkeitsaussagen für eine Klasse verallgemeinerter konvexer Funktionen in topologischen linearen Räumen. (German) Publ. Inst. Math. (Beograd) (N.S.) 23 (37), pp. 13-20, (1978). 
[9] W. W. Breckner and G. Orbán, Continuity properties of rationally s-convex mappings with values in an ordered topological linear space. Universitatea "Babeş-Bolyai, Facultatea de Matematica, Cluj-Napoca, viii+92, (1978).

[10] P. Cerone and S. S. Dragomir, Midpoint-type rules from an inequalities point of view, Ed. G. A. Anastassiou, Handbook of AnalyticComputational Methods in Applied Mathematics, CRC Press, New York., pp. 135-200, (2000).

[11] P. Cerone and S. S. Dragomir, New bounds for the three-point rule involving the Riemann-Stieltjes integrals, in Advances in Statistics Combinatorics and Related Areas, C. Gulati, et al. (Eds.), World Science Publishing, pp. 53-62, (2002).

[12] P. Cerone, S. S. Dragomir and J. Roumeliotis, Some Ostrowski type inequalities for $n$-time differentiable mappings and applications, Demonstratio Mathematica, 32 (2), pp. 697-712, (1999).

[13] G. Cristescu, Hadamard type inequalities for convolution of $h$-convex functions. Ann. Tiberiu Popoviciu Semin. Funct. Equ. Approx. Convexity 8, pp. 3-11, (2010).

[14] S. S. Dragomir, Some remarks on Hadamard's inequalities for convex functions, Extracta Math., 9 (2), pp. 88-94, (1994).

[15] S. S. Dragomir, Refinements of the Hermite-Hadamard integral inequality for log-convex functions, Austral. Math. Soc. Gaz. 28, No. 3, pp. 129-134, (2001).

[16] S. S. Dragomir, Ostrowski's inequality for monotonous mappings and applications, J. KSIAM, 3 (1), pp. 127-135, (1999).

[17] S. S. Dragomir, The Ostrowski's integral inequality for Lipschitzian mappings and applications, Comp. Math. Appl., 38, pp. 33-37, (1999).

[18] S. S. Dragomir, On the Ostrowski's inequality for Riemann-Stieltjes integral, Korean J. Appl. Math., 7, pp. 477-485, (2000).

[19] S. S. Dragomir, On the Ostrowski's inequality for mappings of bounded variation and applications, Math. Ineq. 83 Appl., 4 (1), pp. 33-40, (2001). 
[20] S. S. Dragomir, On the Ostrowski inequality for Riemann-Stieltjes integral $\int_{a}^{b} f(t) d u(t)$ where $f$ is of Hölder type and $u$ is of bounded variation and applications, J. KSIAM, 5 (1), pp. 35-45, (2001).

[21] S. S. Dragomir, Ostrowski type inequalities for isotonic linear functionals, J. Inequal. Pure $\mathscr{E}$ Appl. Math., 3(5), Art. 68, (2002).

[22] S. S. Dragomir, An inequality improving the first Hermite-Hadamard inequality for convex functions defined on linear spaces and applications for semi-inner products. J. Inequal. Pure Appl. Math. 3, No. 2, Article 31, 8, (2002).

[23] S. S. Dragomir, An inequality improving the first Hermite-Hadamard inequality for convex functions defined on linear spaces and applications for semi-inner products, J. Inequal. Pure and Appl. Math., 3 (2), Art. 31, (2002).

[24] S. S. Dragomir, An inequality improving the second HermiteHadamard inequality for convex functions defined on linear spaces and applications for semi-inner products, J. Inequal. Pure and Appl. Math., 3 (3), Art. 35, (2002).

[25] S. S. Dragomir, An Ostrowski like inequality for convex functions and applications, Revista Math. Complutense, 16 (2), pp. 373-382, (2003).

[26] S. S. Dragomir, Operator Inequalities of Ostrowski and Trapezoidal Type. Springer Briefs in Mathematics. Springer, New York, (2012). $\mathrm{x}+112$ pp. ISBN: $978-1-4614-1778-1$

[27] S. S. Dragomir, Some new inequalities of Hermite-Hadamard type for GA-convex functions, Preprint RGMIA Res. Rep. Coll. 18 (2015), Art 33. [http://rgmia.org/papers/v18/v18a33.pdf].

[28] S. S. Dragomir, New inequalities of Hermite-Hadamard type for logconvex functions, Preprint RGMIA Res. Rep. Coll. 18, (2015), Art 42. [http://rgmia.org/papers/v18/v18a42.pdf].

[29] S. S. Dragomir, P. Cerone, J. Roumeliotis and S. Wang, A weighted version of Ostrowski inequality for mappings of Hölder type and applications in numerical analysis, Bull. Math. Soc. Sci. Math. Romanie, 42(90) (4), pp. 301-314, (1999). 
[30] S. S. Dragomir and S. Fitzpatrick, The Hadamard inequalities for sconvex functions in the second sense. Demonstratio Math. 32, No. 4, pp. 687-696, (1999).

[31] S. S. Dragomir and S. Fitzpatrick, The Jensen inequality for s-Breckner convex functions in linear spaces. Demonstratio Math. 33, No. 1, pp. 43-49, (2000).

[32] S. S. Dragomir and B. Mond, Integral inequalities of Hadamard's type for log-convex functions, Demonstratio Math., 31 (2), pp. 354-364, (1998).

[33] S. S. Dragomir and C. E. M. Pearce, On Jensen's inequality for a class of functions of Godunova and Levin. Period. Math. Hungar., 33, No. 2, pp. 93-100, (1996).

[34] S. S. Dragomir and C. E. M. Pearce, Quasi-convex functions and Hadamard's inequality, Bull. Austral. Math. Soc. 57, pp. 377-385, (1998).

[35] S. S. Dragomir and C. E. M. Pearce, Selected Topics on HermiteHadamard Inequalities and Applications, RGMIA Monographs, Victoria University, (2000).

[36] S. S. Dragomir, J. Pečarić and L. Persson, Some inequalities of Hadamard type. Soochow J. Math. 21, No. 3, pp. 335-341, (1995).

[37] S. S. Dragomir and Th. M. Rassias (Eds), Ostrowski Type Inequalities and Applications in Numerical Integration, Kluwer Academic Publisher, (2002).

[38] S. S. Dragomir and S. Wang, A new inequality of Ostrowski's type in $L_{1}-$ norm and applications to some special means and to some numerical quadrature rules, Tamkang J. of Math., 28, pp. 239-244, (1997).

[39] S. S. Dragomir and S. Wang, Applications of Ostrowski's inequality to the estimation of error bounds for some special means and some numerical quadrature rules, Appl. Math. Lett., 11 , pp. 105-109, (1998).

[40] S. S. Dragomir and S. Wang, A new inequality of Ostrowski's type in $L_{p}$-norm and applications to some special means and to some numerical quadrature rules, Indian J. of Math., 40 (3), pp. 245-304, (1998). 
[41] A. El Farissi, Simple proof and refeinment of Hermite-Hadamard inequality, J. Math. Ineq. 4, No. 3, pp. 365-369, (2010).

[42] L. Fejér, Über die Fourierreihen, II, Math. Naturwiss. Anz Ungar. Akad. Wiss. 24, pp. 369-390, (1906). (In Hungarian).

[43] P. M. Gill, C. E. M. Pearce and J. Pečarić, Hadamard's inequality for rconvex functions, Journal of Mathematical Analysis and Applications. 215, No. 2, pp. 461-470, (1997).

[44] E. K. Godunova and V. I. Levin, Inequalities for functions of a broad class that contains convex, monotone and some other forms of functions. (Russian) Numerical mathematics and mathematical physics (Russian), 138-142, 166, Moskov. Gos. Ped. Inst., Moscow, (1985).

[45] H. Hudzik and L. Maligranda, Some remarks on s-convex functions. Aequationes Math. 48, No. 1, pp. 100-111, (1994).

[46] E. Kikianty and S. S. Dragomir, Hermite-Hadamard's inequality and the p-HH-norm on the Cartesian product of two copies of a normed space, Math. Inequal. Appl. Mathematical Inequalities Applications, Volume 13, Number 1, pp. 1-32, (2010).

[47] U. S. Kirmaci, M. Klaričić Bakula, M. E Özdemir and J. Pečarić, Hadamard-type inequalities for s-convex functions. Appl. Math. Comput. 193, No. 1, pp. 26-35, (2007).

[48] M. A. Latif, On some inequalities for h-convex functions. Int. J. Math. Anal. (Ruse) 4, No. 29-32, pp. 1473-1482, (2010).

[49] D. S. Mitrinović and I. B. Lacković, Hermite and convexity, Aequationes Math. 28, pp. 229-232, (1985).

[50] D. S. Mitrinović and J. E. Pečarić, Note on a class of functions of Godunova and Levin. C. R. Math. Rep. Acad. Sci. Canada 12, No. 1, pp. 33-36, (1990).

[51] M. A. Noor, K. I. Noor and M. U. Awan, Some inequalities for geometrically-arithmetically $h$-convex functions, Creat. Math. Inform. 23, No. 1, 91-98, (2014).

[52] C. E. M. Pearce and A. M. Rubinov, P-functions, quasi-convex functions, and Hadamard-type inequalities. J. Math. Anal. Appl. 240, No. 1, pp. 92-104, (1999). 
[53] J. E. Pečarić and S. S. Dragomir, On an inequality of Godunova-Levin and some refinements of Jensen integral inequality. Itinerant Seminar on Functional Equations, Approximation and Convexity (Cluj-Napoca, 263-268, Preprint, 89-6, Univ. "Babeş-Bolyai, Cluj-Napoca, (1989).

[54] J. Pečarić and S. S. Dragomir, A generalization of Hadamard's inequality for isotonic linear functionals, Radovi Mat. (Sarajevo) 7, pp. 103-107, (1991).

[55] M. Radulescu, S. Radulescu and P. Alexandrescu, On the GodunovaLevin-Schur class of functions. Math. Inequal. Appl. 12, No. 4, pp. 853-862, (2009).

[56] M. Z. Sarikaya, A. Saglam, and H. Yildirim, On some Hadamard-type inequalities for h-convex functions. J. Math. Inequal. 2, No. 3, pp. 335-341, (2008).

[57] E. Set, M. E. Özdemir and M. Z. Sarıkaya, New inequalities of Ostrowski's type for s-convex functions in the second sense with applications. Facta Univ. Ser. Math. Inform. 27, No. 1, pp. 67-82, (2012).

[58] M. Z. Sarikaya, E. Set and M. E. Özdemir, On some new inequalities of Hadamard type involving h-convex functions. Acta Math. Univ. Comenian. (N.S.) 79, No. 2, pp. 265-272, (2010).

[59] W. T. Sulaiman, Refinements to Hadamard's inequality for log-convex functions. Applied Mathematics, 2, pp. 899-903, (2011).

[60] M. Tunç, Ostrowski-type inequalities via h-convex functions with applications to special means. J. Inequal. Appl., 326, (2013).

[61] S. Varošanec, On h-convexity. J. Math. Anal. Appl. 326, No. 1, pp. 303-311, (2007).

[62] X.-M. Zhang, Y.-M. Chu and X.-H. Zhang, The Hermite-Hadamard type inequality of GA-convex functions and its application, Journal of Inequalities and Applications, Volume, Article ID 507560, 11 pages, (2010). 


\section{S. S. Dragomir}

Mathematics,

College of Engineering \& Science

Victoria University,

P. O. Box 14428

Melbourne City, MC 8001,

Australia

e-mail : sever.dragomir@vu.edu.au 